\title{
p190RhoGAP and Rap-dependent RhoGAP (ARAP3) inactivate RhoA in response to nerve growth factor leading to neurite outgrowth from PC12 cells
}

\author{
Chan-Young Jeon, Hee-Jun Kim, Jae-Yong Lee, \\ Jaebong Kim, Sung-Chan Kim and \\ Jae-Bong Park ${ }^{1}$ \\ Department of Biochemistry \\ Hallym University College of Medicine \\ Chuncheon 200-702, Korea \\ ${ }^{1}$ Corresponding author: Tel, 82-33-248-2542; \\ Fax, 82-33-244-8425; E-mail, jbpark@ hallym.ac.kr \\ DOI 10.3858/emm.2010.42.5.035
}

Accepted 26 February 2010

Available Online 4 March 2010

Abbreviations: ARAP3, Rap-dependent RhoGAP; FGF, fibroblast growth factor; FRS-2, FGF receptor substrate-2; GAP, GTPase activating proteins; GEF, guanine nucleotide exchange factor; GSTRhotekin-RBD, GST-Rhotekin Rho binding domain; His-RalGDS$R B D$, His-Ral guanine nucleotide dissociation stimulator-Rap binding domain; LPA, lysophosphatidic acid; NGF, nerve growth factor; ROCK, Rho-dependent kinase

\begin{abstract}
Rat pheochromocytoma (PC12) cells have been used to investigate neurite outgrowth. Nerve growth factor (NGF) has been well known to induce neurite outgrowth from PC12 cells. RhoA belongs to Ras-related small GTP-binding proteins, which regulate a variety of cellular processes, including cell morphology alteration, actin dynamics, and cell migration. NGF suppressed GTP-RhoA levels after $12 \mathrm{~h}$ in PC12 cells and was consistently required for a long time to induce neurite outgrowth. Constitutively active (CA)-RhoA suppressed neurite outgrowth from $\mathrm{PC} 12$ cells in response to NGF, whereas dominant-negative (DN)-RhoA stimulated it, suggesting that RhoA inactivation is essential for neurite outgrowth. Here, we investigated the mechanism of RhoA inactivation. DN-p190RhoGAP abrogated neurite outgrowth, whereas wild-type (WT)-p190RhoGAP and WT-Src synergistically stimulated it along with accelerating RhoA inactivation, suggesting that p190RhoGAP, which can be activated by $\mathrm{Src}$, is a major component in inhibiting RhoA in response to NGF in PC12 cells. Contrary to RhoA, Rap1 was activated by NGF, and DN-Rap1 suppressed neu-
\end{abstract}

rite outgrowth, suggesting that Rap1 is also essential for neurite outgrowth. RhoA was co-immunoprecipitated with Rap1, suggesting that Rap1 interacts with RhoA. Furthermore, a DN-Rap-dependent RhoGAP (ARAP3) prevented RhoA inactivation, abolishing neurite formation from $P C 12$ cells in response to NGF. These results suggest that NGF activates Rap1, which, in turn, up-regulates ARAP3 leading to RhoA inactivation and neurite outgrowth from PC12 cells. Taken together, p190RhoGAP and ARAP3 seem to be two main factors inhibiting RhoA activity during neurite outgrowth in PC12 cells in response to NGF.

Keywords: ARAP3 protein; nerve growth factor; neurites; p190RhoGAP; PC12 cells; Rap1; RhoA

\section{Introduction}

Nerve growth factor (NGF) is best known as a molecule that promotes the survival and differentiation of sensory and sympathetic neurons. Studies on the intracellular signaling cascades triggered by NGF have depended heavily on in vitro models using primary cultures or the rat pheochromocytoma cell line (PC12) (Sofroniew et al., 2001). NGF also induces differentiation of PC12 cells, expressed as neurite outgrowths. NGF binds to two receptors, TrkA and p75 ${ }^{\mathrm{NTR}}$ : TrkA is a receptor tyrosine kinase (RTK), and p75 ${ }^{\mathrm{NTR}}$ is a transmembrane glycoprotein that binds all members of the neurotrophin family. In the stimulating process by neurotrophins, activation of TrkA signaling is sufficient for eliciting neurite outgrowth of PC12 cells, and another NGF receptor, p $75^{\text {NTR }}$ signaling plays a modulatory role in the formation of fine synaptic bouton-like structures (Lad et al., 2003). However, $p 75^{\mathrm{NTR}}$ alters the conformation of TrkA to generate high-affinity NGF binding (Esposito et al., 2001). In the NGF-promoted signaling response, sustained activation of ERK1/2 is essential for neurite outgrowth in PC12 cells (York et al., 1998; Kao et al., 2001).

The Ras-related Rho GTPases are molecular switches that regulate diverse cellular processes, including cell proliferation, differentiation, migration, transcription, and actin dynamics (Bishop and Hall, 2000). Activity of Rho GTPases is regulated by 
A

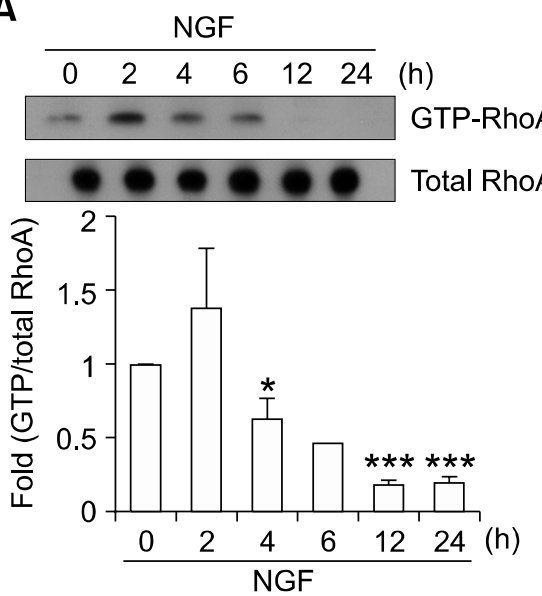

\section{C}

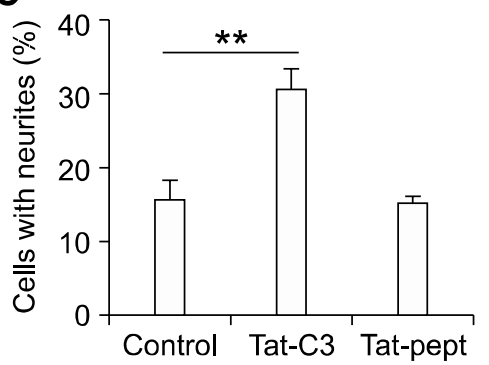

D

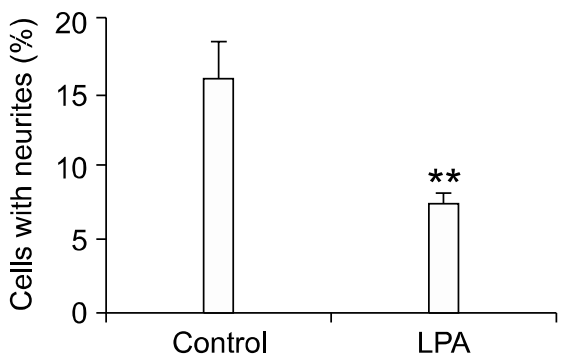

$\mathbf{F}$

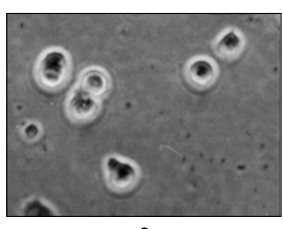

0

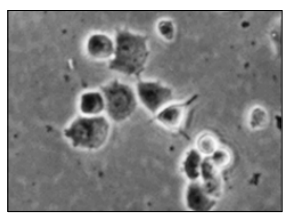

8

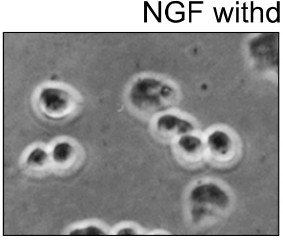

0.5

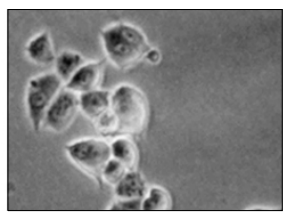

16

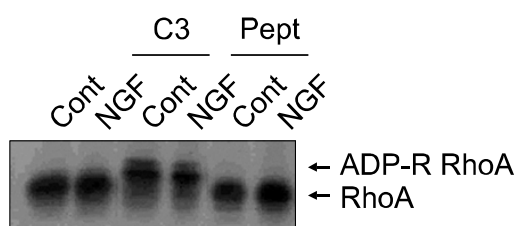

E

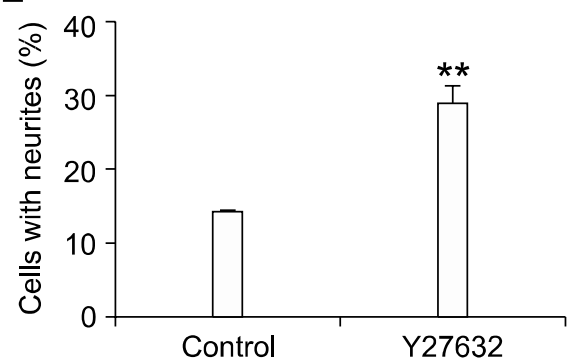

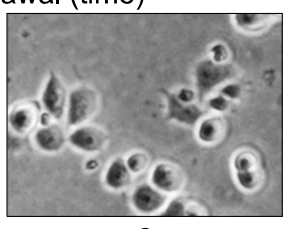

2

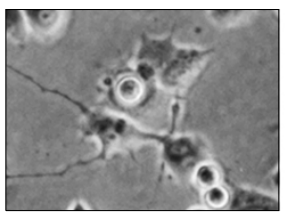

32

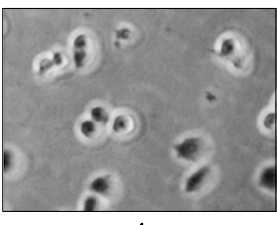

4

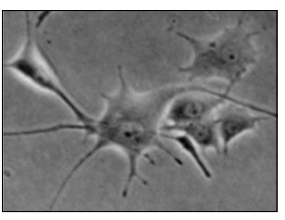

Figure 1. NGF reduces GTP-RhoA levels in PC12 cells. (A) PC12 cells were treated with $100 \mathrm{ng} / \mathrm{ml}$ NGF for the indicated times at $37^{\circ} \mathrm{C}$ and lysed. The cell lysates were incubated with the GST-Rhotekin-RBD domain for GTP-RhoA detection. Bound proteins were analyzed by Western blotting. (B) Wild-type (WT)-RhoA, constitutively active (CA)-RhoA (V14), or dominant negative (DN)-RhoA (N19) cDNAs were co-transfected with a green fluorescence protein (GFP) plasmid and incubated in serum-containing media for $24 \mathrm{~h}$. The transfected PC12 cells were split and incubated for 12 $h$ in serum-starved media, and the cells were then treated with 100 $\mathrm{ng} / \mathrm{ml}$ NGF for 2 days to determine induction of neurites. GFP-positive cells were counted under a microscope (Zeiss, Gottingen, Germany). Transfected HA-RhoA was identified using a Western blot (lower panel). (C) PC12 cells were pre-treated without (Control) or with $0.33 \mu \mathrm{M}$ Tat-C3 toxin and $0.33 \mu \mathrm{M}$ Tat-peptide for $1 \mathrm{~h}$ and then treated with 100 $\mathrm{ng} / \mathrm{ml}$ NGF for 2 days. Modified RhoA by Tat-C3 in PC12 cells was determined by Western blotting with anti-RhoA antibody (right panel). (D) PC12 cells were preincubated for 1 $\mathrm{h}$ with $10 \mu \mathrm{M}$ lysophosphatidic acid (LPA) and then treated with 100 $\mathrm{ng} / \mathrm{ml}$ NGF for 2 days, and the cells with neurites were counted. (E) PC12 cells were pre-incubated with $10 \mu \mathrm{M}$ Y27632, a ROCK inhibitor, for $30 \mathrm{~min}$, then treated with 100 $\mathrm{ng} / \mathrm{ml}$ NGF for 2 days, and cells harboring neurites were counted. Data are presented as means \pm S.E. of three independent experiments $\left({ }^{*} P\right.$ $<0.05 ;{ }^{* *} P<0.01$; ${ }^{* * *} P<0.001$, compared with control using student's $t$-test). (F) PC12 cells were incubated for $12 \mathrm{~h}$ in serum-starved media, then treated with $100 \mathrm{ng} / \mathrm{ml}$ NGF for the indicated times, and then washed out. The cells were incubated with fresh serum-starved media for up to 4 days to induce 96 (h) neurite outgrowth. several factors. The GDP-bound form becomes activated through the action of guanine nucleotide exchange factors (GEFs), whereas the intrinsic GTPase activity of Rho proteins is accelerated by
GTPase activating proteins (GAPs) (Luo, 2000). In the unstimulated state, Rho and Rac can be associated with RhoGDI (guanine nucleotide dissociation inhibitor). RhoGDI seems to sequester 
GDP-bound Rho GTPases in the cytoplasm, inhibiting their spontaneous GDP/GTP exchange activity (Bishop and Hall, 2000). RhoA induces stress fibers composed of filamentous actin and activation of the myosin light chain by phosphorylation, leading to cell contraction (Bishop and Hall, 2000). Rap was identified as converting the Ras-transformed cells. Rap mediates ERK activation through the binding of B-Raf, a close relative of Raf1 (Bos et al., 2003). In particular, Rap1 is activated by NGF in PC12 cells, which prolongs ERK activation, leading to neurite outgrowth from PC12 cells (Kao et al., 2001; Wu et al., 2001).

It has been reported that Rho inactivation is essential for neuronal morphogenesis (Luo, 2000; da Silva and Dotti, 2002). In particular, NGF inactivates RhoA in PC12 cells, which is closely relevant to neurite outgrowth from PC12 cells (Wu et al., 2001; Yamaguchi et al., 2001; Nusser et al., 2002). However, they observed a short transient inactivation of RhoA by NGF. Furthermore, it is not clearly elucidated how RhoA is inactivated in PC12 cells in response to NGF. Among the numerous RhoGAPs, p190RhoGAP exists universally in various cells (Brouns et al., 2000). Although it was reported that $p 190$ RhoGAP induces axonal outgrowth and branching (Billuart et al., 2001; Brouns et al., 2001), it was not clearly elucidated whether p190RhoGAP is responsible for the signals of NGF in PC12 cells until now. The function of Rap1 on RhoA activity during neurite outgrowth of PC12 cells was not evident either.

In this study, we describe that RhoA was inactivated and remained inactive for an extended time during neurite outgrowth from PC12 cells, which was mediated by p190RhoGAP in response to NGF. In addition, the Rap1-dependent RhoGAP ARAP3 that was activated by GTP-Rap1 inactivated RhoA in PC12 cells along with neurite outgrowth upon exposure to NGF.

\section{Results}

\section{NGF reduced GTP-RhoA levels of PC12 cells}

To confirm the involvement RhoA in NGF signaling, the activity of RhoA in response to NGF was determined by measuring GTP-RhoA using a pull-down assay with GST-Rhotekin-RBD beads. NGF significantly reduced GTP-RhoA levels in PC12 cells after 4-6 $\mathrm{h}$ of administration, and the levels reached minimal values at $12-24 \mathrm{~h}$ (Figure 1A). The GTP-RhoA level at $2 \mathrm{~h}$ seemed to be augmented, but it was not significant. This indicates that RhoA inactivation is related to the neurite outgrowth of PC12 cells induced by NGF. In order to further examine the role of RhoA in neurite outgrowth from PC12 cells in response to NGF, the effects of cDNAs of RhoA were examined. Transiently transfected CA-RhoA (RhoA V14) significantly inhibited neurite outgrowth of PC12 cells in response to NGF, whereas transfected DN-RhoA (RhoA N19) stimulated it (Figure 1B), suggesting that RhoA inactivation stimulates neurite outgrowth of PC12 cells in response to NGF. Moreover, Tat-C3 toxin markedly enhanced neurite outgrowth of PC12 cells induced by NGF (Figure 1C). Tat-C3 retarded the gel mobility of endogenous RhoA, probably by modification of ADP-ribosylation, suggesting that the Tat-C3 toxin was active. In contrast, LPA, known as a RhoA activator, decreased the extent of neurite outgrowth in response to NGF (Figure 1D), which was consistent with the published result on NGF (Kozma et al., 1997). In addition, Y27632, a pharmacologic inhibitor of Rho-dependent kinase (ROCK), stimulated neurite outgrowth of PC12 cells in response to NGF, suggesting that ROCK is a downstream effector protein regulating neurite outgrowth (Figure 1E). To determine the length of NGF stimulation required to induce neurite outgrowth from PC12 cells, we washed out NGF from serum-starved media after the defined period and then incubated the cells in media containing a normal serum concentration without NGF. Withdrawal of NGF from the media at an early period up to $16 \mathrm{~h}$ rarely exhibited neurites above two cell body lengths (Figure 1F), suggesting that neurite induction of PC12 cells requires continuous stimulation of NGF for a long time, above $32 \mathrm{~h}$.

\section{p190RhoGAP and Src are implicated in the RhoA inactivation of PC12 cells by NGF}

To understand how RhoA is inactivated by NGF stimulation, we examined whether p190RhoGAP inactivates RhoA upon exposure to NGF during neurite outgrowth of PC12 cells because p190RhoGAP is known to be most abundant RhoGAP in neural tissues (Brouns et al., 2000). Transient transfection of DN-p190RhoGAP, which was deficient in the N-terminal GTP-binding domain, significantly attenuated neurite outgrowth of PC12 cells in response to NGF, whereas WT-p190RhoGAP did not (Figure 2A). Since it is known that Src activates p190RhoGAP by phosphorylation (Chang et al., 1995; Haskell et al., 2001), we examined a role for Src in neurite outgrowth of PC12 cells. Treatment with the Src inhibitors PP2 and SU6656 markedly suppressed neurite outgrowth of PC12 cells, whereas PP3, a control molecule for PP2 that does not inhibit Src, did not significantly reduce 
A

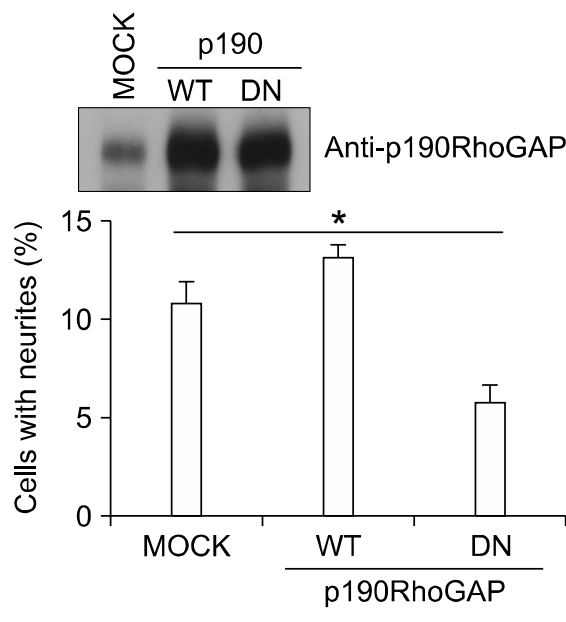

\section{C}

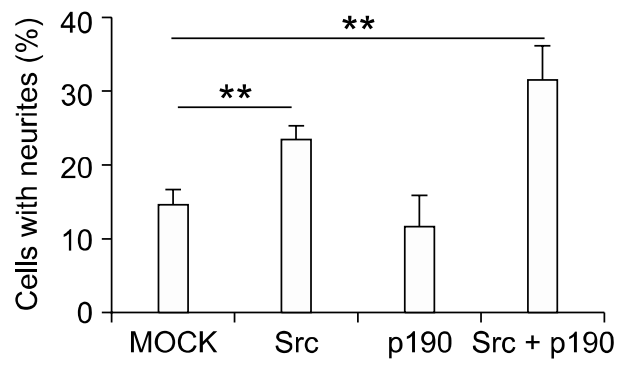

D

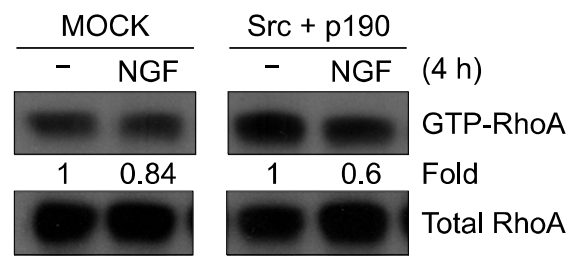

B
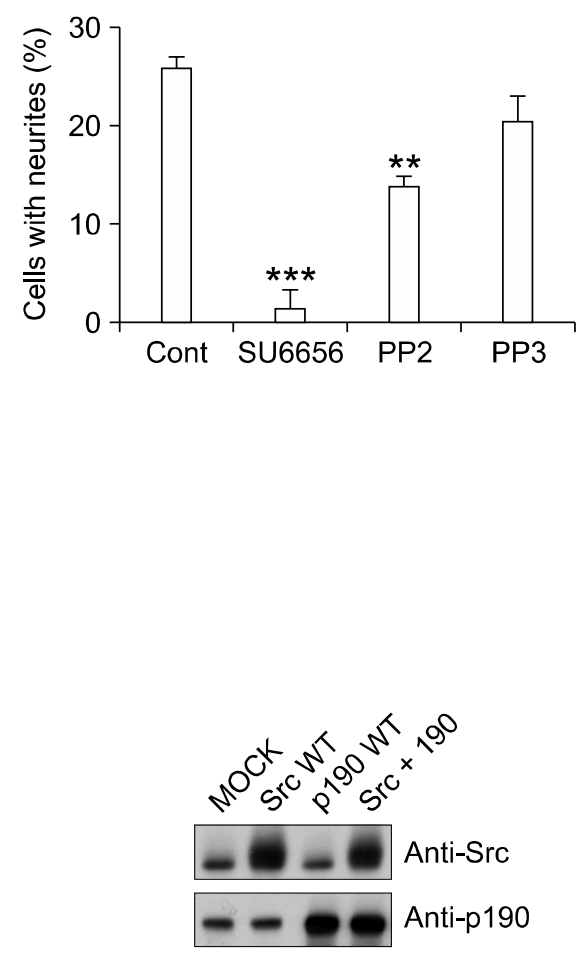

E

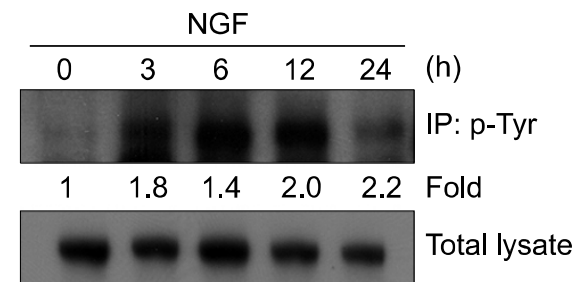

IB: p190RhoGAP

Figure 2. p190RhoGAP and Src are implicated in neurite outgrowth of PC12 cells in response to NGF. (A) PC12 cells were transiently co-transfected with GFP and control (MOCK), WT- or DN-p190RhoGAP (lacking the GTP-binding domain) plasmid and incubated in serum-containing media for $24 \mathrm{~h}$. The transfected PC12 cells were serum-starved for $12 \mathrm{~h}$ and then treated with $100 \mathrm{ng} / \mathrm{ml} \mathrm{NGF}$ for 2 days. Cells with neurites were assessed. Expression of transfected p190RhoGAP was identified using a Western blot in the upper panel. Data are represented as means \pm S.E. of three independent experiments $\left({ }^{*} P<0.05\right.$, compared with control using Student's t-test). (B) PC12 cells were pretreated with $20 \mu \mathrm{M}$ SU6656 (a Src family kinase inhibitor), $20 \mu \mathrm{M}$ PP2 (an inhibitor of the Src family of tyrosine kinases), or $20 \mu \mathrm{M} \mathrm{PP3} \mathrm{(a} \mathrm{control} \mathrm{compound} \mathrm{for} \mathrm{PP2)} \mathrm{for} 30 \mathrm{~min}$, and $100 \mathrm{ng} / \mathrm{ml}$ NGF was then administered for 2 days. The cells with neurites were counted. Data are represented as means \pm S.E. of three independent experiments $\left({ }^{* *} P<0.01 ;{ }^{* * *} P<0.001\right.$, compared with control using Student's t-test). (C) PC12 cells were transiently co-transfected with GFP and MOCK, WT-c-Src, WT-p190RhoGAP, or WT-c-Src plus WT-p190RhoGAP plasmid and incubated in serum-containing media for $24 \mathrm{~h}$. The transfected PC12 cells were serum-starved for $12 \mathrm{~h}$ and treated with $100 \mathrm{ng} / \mathrm{ml}$ NGF for 2 days. GFP-positive cells with neurites were counted. Expression of transfected Src and p190RhoGAP was identified in the right panel. Data were presented as means \pm S.E. of three independent experiments $\left({ }^{* *} P<0.01\right.$, compared with control using Student's $t$-test). (D) Either control (MOCK) or WT-c-Src plasmid and WT-p190RhoGAP were transiently overexpressed in PC12 cells and incubated in serum-containing media for $24 \mathrm{~h}$. After serum starvation, the cells were treated with $100 \mathrm{ng} / \mathrm{ml}$ NGF for $4 \mathrm{~h}$. GTP-loaded RhoA was analyzed using the GST-pull down assay as described in methods. The amount of RhoA pulled down with Rhotekin-RBD or total lysate was visualized by immunoblotting using anti-RhoA antibody. Data are representative of two independent experiments. (E) PC12 cells were treated with $100 \mathrm{ng} / \mathrm{ml}$ NGF for indicated times and lysed. p190RhoGAP was immunoprecipitated from cell lysates with anti-phospho-tyrosine antibody and was analyzed by Western blot using anti-p190RhoGAP antibody to assess tyrosine phosphorylation. Data are representative of three independent experiments demonstrating the same patterns. 
A

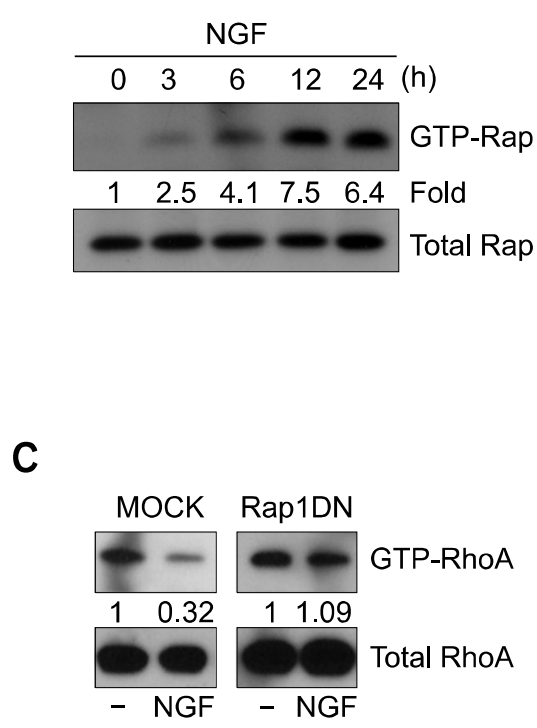

B

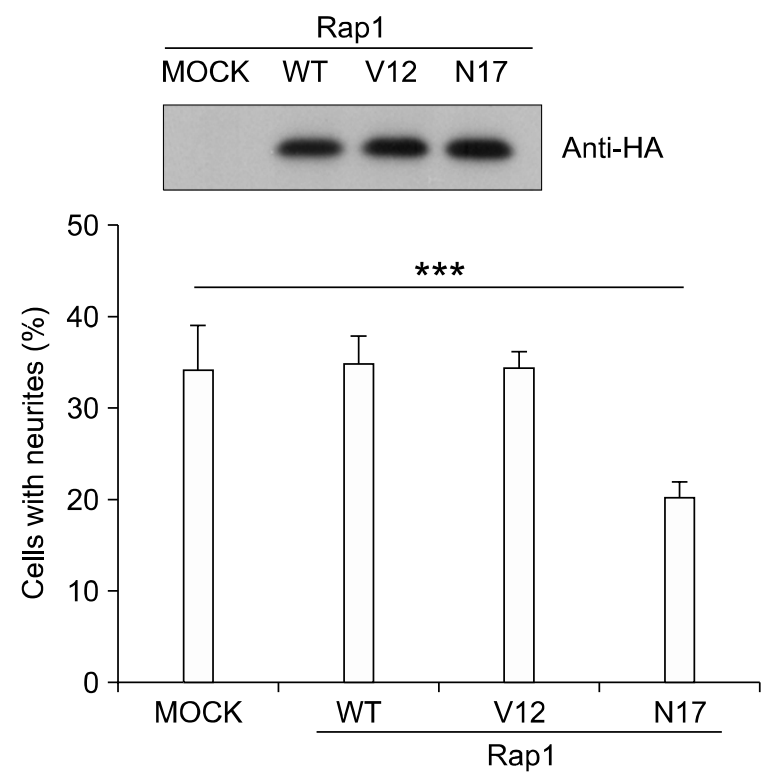

Figure 3. Rap1 is involved in neurite outgrowth of PC12 cells in response to NGF. (A) Active Rap1 was measured in PC12 cells in response to NGF using a pull-down assay with the His-RalGDS-RBD domain, and the level of the GTP-bound Rap1 was visualized by immunoblotting using anti-Rap1 antibody. Data are representative of three independent experiments. (B) PC12 cells were transiently co-transfected with GFP and control (MOCK), WT-, CA (V14) -, or DN (N19)-Rap1 plasmid and incubated with serum-containing media for $24 \mathrm{~h}$. The transfected PC12 cells were split and incubated for $12 \mathrm{~h}$ with serum-starved media. The cells were treated with $100 \mathrm{ng} / \mathrm{ml} \mathrm{NGF}$ for 2 days for induction of neurites, and cells with neurites were determined. Expression of transfected HA-Rap1 was identified with Western blot using anti-HA antibody (upper panel). Data are represented as means \pm S.E. of three independent experiments ( ${ }^{* * *} P<0.001$, compared with control using Student's $t$-test). (C) PC12 cells were transfected with control plasmid (MOCK) or DN-Rap1 and incubated in serum-containing media for $24 \mathrm{~h}$. The cells were incubated in serum-starved media for $12 \mathrm{~h}$ and then treated with $100 \mathrm{ng} / \mathrm{ml} \mathrm{NGF}$ for $24 \mathrm{~h}$. GTP-RhoA was analyzed using the GST-pull down assay. The amount of RhoA pulled down with Rhotekin-RBD or total lysate was visualized by immunoblotting. Data are representative of three independent experiments with similar results.

neurite outgrowth, suggesting that $\mathrm{Src}$ is also involved in neurite outgrowth of PC12 cells in response to NGF (Figure 2B). Consistently, transfection of $\mathrm{c}-\mathrm{Src}$ alone augmented increased neurite outgrowth from PC12 cells (Figure $2 \mathrm{C}$ ). Moreover, co-transfection of WT-c-Src and WT-p190RhoGAP synergistically greatly enhanced the neurite outgrowth of PC12 cells in response to NGF (Figure 2C). As a consequence of co-transfection with c-Src and p190RhoGAP cDNAs, GTP-RhoA levels in cells were lower than levels shown in control cells in response to NGF (Figure 2D). Thus, we examined whether p190RhoGAP was phosphorylated in response to NGF. p190RhoGAP was first pulled-down with anti-phospho-Tyr antibody (4G10) and p190RhoGAP was detected by western blot: the p190RhoGAP immunoprecipitated with anti-phospho-Tyr antibody was increased, indicating that NGF increases tyrosine phosphorylation of p190RhoGAP (Figure 2E). It is likely that NGF activates p190RhoGAP by phosphorylation, probably via c-Src.

\section{Rap1 is also involved in neurite outgrowth of PC12 cells induced by NGF}

Although it is well known that Rap1 is involved in the differentiation of $\mathrm{PC} 12$ cells in response to NGF, which induces sustained activation of ERK1/2 through B-Raf (York et al., 1998), it was controversial that Rap1 was involved in neurite outgrowth of PC12 cells in response to NGF (Vossler et al., 1997; Hisata et al., 2007). Here, we evaluated GTP-Rap1 levels up to $24 \mathrm{~h}$ in PC12 cells in response to NGF, although a previous document showed GTP-Rap1 levels for an early period within $3 \mathrm{~h}$ in response to NGF (Wu et al., 2001). We found that NGF augmented GTP-Rap1 levels in PC12 cells after $3 \mathrm{~h}$ and sustained the levels for at least $24 \mathrm{~h}$ (Figure $3 \mathrm{~A}$ ). To confirm whether Rap1 was relevant to the regulation of neurite outgrowth, the effects of transfection of Rap1 cDNA constructs were assessed. Transfection of DN-Rap1 (Rap1N17) significantly inhibited neurite outgrowth, whereas WT- and CA-Rap1 (Rap1 V12) did not affect it in response to NGF (Figure 3B). A putative inhibitor of Rap1, 8CPT-cGMP (Jenei et al., 2006), significantly 
A

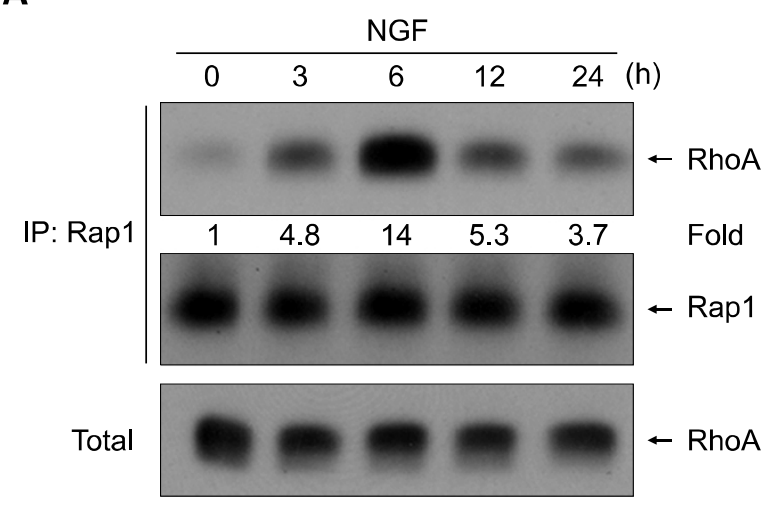

C

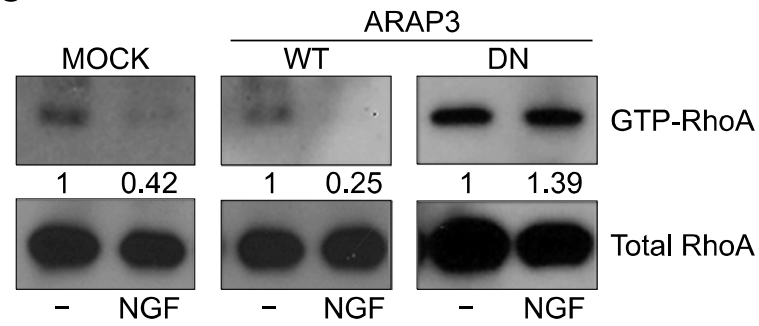

B

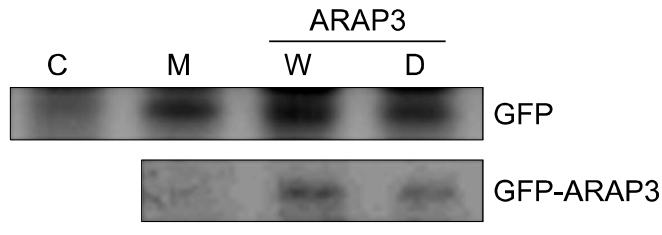

WB: anti-GFP

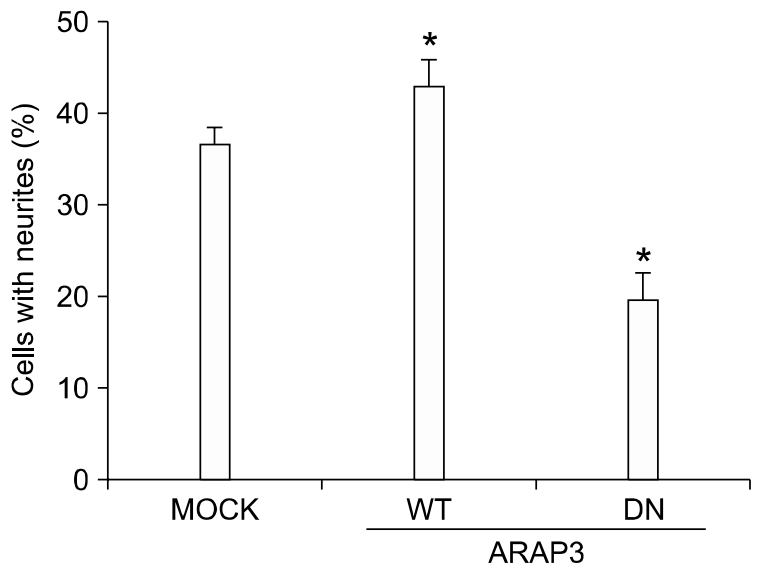

Figure 4. ARAP3 is a mediator of RhoA inactivation. (A) The interaction of Rap1 and RhoA in response to NGF was examined. Serum-starved PC12 cells were treated with $100 \mathrm{ng} / \mathrm{ml} \mathrm{NGF}$ for various times as indicated and lysed. The lysates were immunoprecipitated with anti-Rap1 antibody. The interaction was analyzed by Western blot using anti-RhoA antibody. Data are representative of three independent experiments with similar results. (B) PC12 cells were transiently co-transfected with GFP and control (MOCK), WT-ARAP3 or DN (double mutations in both the RhoGAP and ArfGAP (R982A/C504A) domains)-ARAP3 plasmid and incubated in serum-containing media for $24 \mathrm{~h}$. The transfected PC12 cells were serum-starved for $12 \mathrm{~h}$ and then treated with $100 \mathrm{ng} / \mathrm{ml}$ NGF for 2 days. Cells with neurites were assessed. Expression of GFP-ARAP3 was identified with a Western blot using anti-GFP antibody in the upper panel. Data are represented as means \pm S.E. of three independent experiments ( ${ }^{*}, P<0.05$, compared with control using Student's $t$-test). (C) PC12 cells were transfected with control (MOCK), WT-ARAP3, or DN-ARAP3 plasmid and incubated in serum-containing media for $24 \mathrm{~h}$. The cells were serum-starved for $12 \mathrm{~h}$ and then treated with $100 \mathrm{ng} / \mathrm{ml} \mathrm{NGF}$ for $24 \mathrm{~h}$. GTP-loaded RhoA was analyzed by the GST-pull down assay. The amount of RhoA pulled-down with GST-Rhotekin-RBD or total lysate was visualized by immunoblotting using anti-RhoA antibody. Data are representative of two independent experiments with similar results.

reduced neurite outgrowth in response to NGF (Supplemental Data Figure S1). This suggests that Rap1 is essential for neurite outgrowth. Although it is well known that Rap1 induces sustained activation of ERK for neurite outgrowth of PC12 cells, here, we examined whether Rap1 regulates RhoA activity. In fact, DN-Rap1 cDNA transfection prevented the decrease of GTP-RhoA levels in response to NGF, suggesting that activation of DN-Rap1 cannot inactivate RhoA (Figure 3C).

\section{A Rap-dependent RhoGAP, ARAP3, also plays a role in neurite outgrowth of PC12 cells in response to NGF}

To understand how Rap1 inactivates RhoA, the interaction between RhoA and Rap1 was examined. Rap1 was immunoprecipitated first, and RhoA was then identified with immunoblotting. Co-immunoprecipitated RhoA with Rap1 was in- creased and then diminished depending on NGF treatment time, suggesting that interaction of RhoA with Rap1 is regulated by NGF (Figure 4A). Since it has been documented that Rap1-dependent RhoGAP, ARAP3 can accelerate GTP hydrolysis of RhoA (Krugmann et al., 2004), we examined whether ARAP3 could also inactivate RhoA in PC12 cells upon exposure to NGF. DN-ARAP3, which lacks both RhoGAP and ArfGAP (R982A/C504A) activities, significantly inhibited neurite outgrowth from PC12 cells in response NGF, while WT-ARAP3 slightly increased it (Figure 4B). Consistently, DN-ARAP3 cDNA prevented the decrease of GTP-RhoA levels, whereas WT-ARAP reduced GTP-RhoA levels in response to NGF (Figure 4C). Taken together, these observations suggest that Rap1 activation and subsequent ARAP3 activation induce RhoA inactivation in PC12 cells along with neurite outgrowth in res- 
ponse to NGF.

\section{Discussion}

Although some proposals for the mechanism of RhoA inactivation by a variety of stimuli have been documented (Harada et al., 2005), in this study, we have shown that long-term RhoA inactivation is essential for neurite outgrowth from PC12 cells in response to NGF and that RhoA inactivation is likely to be mediated by p190RhoGAP and a Rap-dependent RhoGAP (ARAP3).

Indeed, NGF started to reduce GTP-RhoA levels after $4 \mathrm{~h}$, reducing it to low basal levels after $12 \mathrm{~h}$, although it seemed to increase GTP-RhoA levels at $2 \mathrm{~h}$, although this was not statistically significant (Figure 1). In some reports, time courses of RhoA inactivation are somehow different: Either RhoA was inactivated in an early period of 3-10 min after treatment of NGF and then recovered to an active state in $1 \mathrm{~h}$ (Yamaguchi et al., 2001), or RhoA was inactivated within $2 \mathrm{~h}$ (Nusser et al., 2002). These studies did not observe GTP-RhoA levels over an extended period in response to NGF. We do not know the reason for this discrepancy. However, it might be due to different conditions for cell culture systems: we maintained or proliferated cells in RPMI to avoid changes in cell morphology, moved cells to DMEM with 1/10-diluted sera for $12 \mathrm{~h}$, and then stimulated cells, which were grown on collagen-coated plates, with NGF. Here, we found that long-term inactivation of RhoA is required for neurite outgrowth because a short-term treatment of NGF and then washing out NGF could not induce neurite outgrowth in $16 \mathrm{~h}$ (Figure 1E). Pretreatment with Tat-C3 toxin markedly enhanced neurite outgrowth (Figure 1C). From these observations, it is inferred that prolonged RhoA inactivation, rather than transient inactivation, may be essential for neurite outgrowth.

Since Src phosphorylates and activates p190RhoGAP (Fincham et al., 1999) and p190RhoGAP regulates axon outgrowth in the brain (Brouns et al., 2001), Src is likely to be a critical regulator of p190RhoGAP and RhoA inactivation in neurite outgrowth from PC12 cells in response to NGF (Figure 2). Src can be activated by dephosphorylation of the Y527 residue by Tyr phosphatases (Roskoski, 2005). One of these phosphatases is Shp2, which is bound to FGF receptor substrate-2 (FRS2) and is involved in the regulation of neurite outgrowth of $\mathrm{PC} 12$ cells in response to FGF (Hadari et al., 1998). In fact, Shp2 regulates Src kinase activity and Ras/Erk activation (Zhang et al., 2004). The docking protein FRS2 binds directly to the NGF receptor, and NGF induces tyrosine phosphorylation of FRS2 (Ong et al., 2000). Thus, the signaling pathway for NGF to inactivate RhoA is likely to be the sequence of 'NGF/TrkA/FRS2/ Shp2/Src/p190RhoGAP/GDP-RhoA'.

Recently, it was controversially documented that si-Rap1 inhibited neurite outgrowth of PC12 cells in response to NGF (Hisata et al., 2007), while DN-Rap1 (N17) does not block neurite outgrowth of PC12 cell in response to NGF (Vossler et al., 1997). However, it is obvious that NGF activates Rap1 in PC12 cells and that Rap1 regulates neurite outgrowth of PC12 cells from our results (Figure 3). We tried to understand how NGF activates Rap1 depending on several published documents. FRS2 phosphorylation provides a scaffold for the assembly of a stable complex of Crk and C3G, an exchange factor activating the small GTPase Rap1 (York et al., 1998). We postulated a signaling pathway of NGF to GDP-RhoA through Rap1 activation as follows: NGF/FRS2/Crk/C3G/Rap1/ARAP3/ GDP-RhoA. Besides C3G, a GEF for Rap1, Rap1-PDZ-GEF1, can also activate Rap1, which induces neurite outgrowth in response to NGF in PC12 cells (Hisata et al., 2007). Thus, Rap1 is likely to be activated through several pathways.

ARAP3 may also be regulated by $\mathrm{PIP}_{3}$ in addition to GTP-Rap1 in PC12 cells. Indeed, NGF augmented p-AKT (Supplemental Data Figure S2), suggesting that $\mathrm{PIP}_{3}$ is produced by NGF. However, we did not investigate the involvement of $\mathrm{PIP}_{3}$ in RhoA inactivation in this study. GTP-Rap1 is able to activate RA-RhoGAP in addition to ARAP3, leading to neurite outgrowth of NG108 cells (Yamada et al., 2005). However, ARAP3 does not exhibit any similarity in amino acid sequence to RA-RhoGAP. This suggests that RhoA may be inactivated through several pathways mediated by active Rap1.

It remains to be elucidated how RhoA inactivation induces neurite formation. In the context of cell morphology, the active RhoA renders cells contracted and rounded (da Silva and Dotti, 2002). However, the active Rac1 and Rac2 cause neurite formation (Daniels et al., 1998; Yasui et al., 2001; Aoki et al., 2005; Welch et al., 2005), although Rac1/Rac2 is essential for regulation of superoxide production (Kim et al., 2008; El-Benna et al., 2009). We also found that NGF induces activation of the Rac1 GTPase (Supplemental Data Figure S3). Furthermore, Rac and Rho GTPases reveal antagonistic actions in the context of neurite outgrowth (Yamaguchi et al., 2001; Nusser et al., 2002). Thus, RhoA should be inactivated to induce Rac activation, which is related to induce neurite 
outgrowth. In conclusion, we demonstrated in this study that RhoA was inactivated by p190RhoGAP and Rap1/ARAP3 along with neurite outgrowth in response to NGF.

\section{Methods}

\section{Materials}

NGF, lysophosphatidic acid (LPA), 8CPT-cGMP, Y27632, aprotinin, pepstatin $A$, sodium orthovanadate, sodium fluoride, poly-L-lysine, bovine serum albumin (BSA) and PD98059 were purchased from Sigma (St. Louis, MO). PP2, PP3, and SU6656 were obtained from Calbiochem (La Jolla, CA). Leupeptin and phenylmethanesulfonyl fluoride (PMSF) were purchased from Boehringer Mannheim (Mannheim, Germany). Rap1 cDNAs were kindly provided by Dr. Hall or obtained from the Missouri S\&T cDNA Resource Center (Rolla, MO). ARAP3 cDNAs were kindly provided by Dr. Krugmann (Babraham Institute, UK). His-RalGDS Rap binding domain (His-RalGDS-RBD) was kindly provided by Dr. Bos (University Medical Center Utrecht, Netherlands). GST-Rhotekin-Rho binding domain (GST-Rhotekin-RBD) was produced from Escherichia coli and the EZ-Detect ${ }^{T M}$ Rho activation kit was purchased from Pierce (Pierce, Rockford, IL). Tat-C3, which is a fusion protein between Tat-peptide and C3 toxin and can readily penetrate into cells leading to inhibition of Rho GTPases, was purified from bacteria containing the Tat-C3 cDNA plasmid using a previously described protocol (Kim et al., 2004). Anti-Rap1, -RhoA, -c-Src, and -actin antibodies were obtained from Santa Cruz Biotechnology (Santa Cruz, CA), anti-p190RhoGAP and -phosphotyrosine (4G10) antibodies were purchased from Upstate (Lake Placid, NY), anti-phospho-ERK, -ERK, -phospho-Akt, and -Akt antibodies were purchased from Cell Signaling Technology (Danvers, MA), anti-green fluorescent protein (GFP) antibody was obtained from BD Biosciences (San Jose, CA) and anti-HA antibody was purchased from Covance (Berkeley, CA).

\section{Cell culture and induction of differentiation}

PC12 cells were cultured in RPMI 1640 medium (Lonza, Walkersville, MD) supplemented with $10 \%$ heat-inactivated horse serum (HS), 5\% heat-inactivated FBS (Lonza), and antibiotics (100 units $/ \mathrm{ml}$ streptomycin and 100 units $/ \mathrm{ml}$ penicillin) (Lonza) at $37^{\circ} \mathrm{C}$ under $5 \% \quad \mathrm{CO}_{2}$ on plates (Corning, Cambridge, MA) coated with collagen type IV. To assess neurite outgrowth, the cells at a density of $5 \times 10^{3}$ cells/well on $60-\mathrm{mm}$ culture dishes were incubated in DMEM medium (Lonza) containing $1 / 10$ serum (1\% HS and $0.5 \%$ FBS: serum-starved medium) and antibiotics. Cells were treated with $100 \mathrm{ng} / \mathrm{ml} \mathrm{NGF}$ in serum-starved medium containing antibiotics and $2 \mathrm{mg} / \mathrm{ml} \mathrm{BSA}$.

\section{Neurite formation assay}

The percentage of neurite-bearing cells was determined by counting at least 100 non-aggregated single cells in at least three arbitrary positions on the dish. A cell was evaluated to be positive for neurite outgrowth if it exhibited neurites with a length at least two times the diameter of the cell body. Generally, cells were visualized using phase contrast (200-fold magnification) on a Nikon Diaphot TMS-F microscope, and images were captured with a Nikon F70 camera using Kodak ISO $400 / 27^{\circ}$ film. For transfection experiments, GFP-positive cells were counted under an Axiovert 200 microscope (Zeiss, Gottingen, Germany) with a 40x/0.10 NA Achiroplan objective and photographed with a Photometrics CoolSNAP.

\section{Transfection}

PC12 cells were transiently co-transfected with $0.2 \mu \mathrm{g}$ of GFP cDNA and $1.8 \mu \mathrm{g}$ of wild-type (WT)-RhoA, constitutively active RhoA (CA)-V14, dominant negative RhoA (DN)-N19, p190RhoGAP (WT or DN: lacking the GTP-binding domain), Src WT, Rap1A (WT, CA-V12, or DN-N17), or ARAP3 (WT or DN: double mutations of RhoGAP and ArfGAP (R982A/C504A)) plasmid using a nucleofector from Amaxa biosystems (Cologne, Germany).

\section{Western blot analysis}

NGF-stimulated PC12 cells were collected, washed once with ice-cold PBS, and lysed with 0.1-0.2 ml RIPA buffer (50 mM Tris- $\mathrm{HCl}, \mathrm{pH} 7.4,150 \mathrm{mM} \mathrm{NaCl}, 1 \%$ Nonidet P-40 (NP-40), 0.25\% NaN3, 1 mM EDTA, 1 mM PMSF, $1 \mu \mathrm{g} / \mathrm{ml}$ aprotinin, $1 \mu \mathrm{g} / \mathrm{ml}$ leupeptin, $1 \mu \mathrm{g} / \mathrm{ml}$ pepstatin $\mathrm{A}, 1 \mathrm{mM}$ sodium orthovanadate, and $1 \mathrm{mM} \mathrm{NaF}$ ). Lysates were centrifuged for $10 \mathrm{~min}$ at $12,000 \times g$ and supernatants were analyzed for protein concentration using a BCA Protein Assay kit (Pierce). Equal amounts of proteins were resolved by SDS-PAGE, transferred to PVDF membranes, and probed with antibodies against RhoA, Rap1A, phospho-tyrosine (4G10), p190RhoGAP, or GFP. Immunoreactive bands were visualized by enhanced chemiluminescence. Bands were scanned using a scanner (HP Scanjet 7400C, Hewlett-Packard), and their intensities were quantified with the Image $\mathrm{J}(\mathrm{NIH})$ program. Statistical analysis was performed with GraphPad Prism4.

\section{Immunoprecipitation}

NGF-stimulated PC12 cells were collected, washed once with ice-cold PBS, and lysed in RIPA buffer. Lysates were centrifuged for $15 \mathrm{~min}$ at $10,000 \times g$, and supernatants were used for binding with anti-phospho-tyrosine or -Rap1 antibodies for $6 \mathrm{~h}$ at $4^{\circ} \mathrm{C}$, after which protein A-conjugated agarose beads (Pierce, Rockford, IL) were added and incubated using a rotating mixer for $2 \mathrm{~h}$ at $4^{\circ} \mathrm{C}$. The beads were washed five times with lysis buffer. The precipitated proteins were eluted with $2 \times$ Laemmli sample buffer by boiling, samples were resolved by electrophoresis on SDS-PAGE, and p190RhoGAP or RhoA was detected with western blotting.

\section{Pull-down assay for activated RhoA and Rap1}

GTP-RhoA levels were detected using the EZ-Detect ${ }^{\mathrm{TM}}$ Rho activation kit (Pierce) according to the manufacturer's 
method, and GTP-Rap1 was assessed using His-RalGDS-RBD, which was expressed in $E$. coli, and bound to Ni-NTA beads. Briefly, growth factor-stimulated PC12 cells were collected, washed once with ice-cold PBS, and lysed in buffer containing $25 \mathrm{mM}$ Tris- $\mathrm{HCl}, \mathrm{pH}$ 7.4, $150 \mathrm{mM} \mathrm{NaCl}, 5 \mathrm{mM} \mathrm{MgCl} 2,1 \% \mathrm{NP}-40,1 \mathrm{mM}$ DTT, $5 \%$ glycerol, $1 \mu \mathrm{g} / \mathrm{ml}$ aprotinin, $1 \mu \mathrm{g} / \mathrm{ml}$ leupeptin and $1 \mathrm{mM}$ PMSF. Samples were incubated on ice for $5 \mathrm{~min}$ followed by centrifugation $\left(16,000 \times \mathrm{g}, 15 \mathrm{~min}, 4^{\circ} \mathrm{C}\right)$. Equivalent amounts of supernatants were incubated with GST-Rhotekin-RBD/GSH-beads for GTP-RhoA, or with His-RalGDS-RBD/Ni-NTA beads for GTP-Rap1 for $12 \mathrm{~h}$ at $4^{\circ} \mathrm{C}$. The beads were washed 5 times with lysis buffer and bound proteins were eluted with $2 \times$ Laemmli sample buffer by boiling. Samples were electrophoresed and analyzed by western blotting with anti-RhoA or anti-Rap1A antibodies.

\section{Supplemental data}

Supplemental Data include three figures and can be found with this article online at http://e-emm.or.kr/article/article_ files/SP-42-5-03.pdf.

\section{Acknowledgements}

We thank Dr. A. Hall for providing Rap1 cDNAs, Dr. J. L. Bos for RalGDS Rap1-binding domain cDNA, and Dr. Krugmann for ARAP3 cDNA constructs. This study was supported by a grant of the Korea Research Foundation (MOEHRD, Basic Research Promotion Fund) (KRF-2008313-E00093).

\section{References}

Aoki K, Nakamura T, Fujikawa K, Matsuda M. Local phosphatidylinositol 3,4,5-trisphosphate accumulation recruits Vav2 and Vav3 to activate Rac1/Cdc42 and initiate neurite outgrowth in nerve growth factor-stimulated PC12 cells. Mol Biol Cell 2005;16:2207-17

Billuart P, Winter CG, Maresh A, Zhao X, Luo L. Regulating axon branch stability: the role of $p 190$ RhoGAP in repressing a retraction signaling pathway. Cell 2001;107:195-207

Bishop AL, Hall A. Rho GTPases and their effector proteins. Biochem J 2000;348:241-55

Bos JL, de Bruyn K, Enserink J, Kuiperij B, Rangarajan S, Rehmann H, Riedl J, de Rooij J, van Mansfeld F, Zwartkruis F. The role of Rap1 in integrin-mediated cell adhesion. Biochem Soc Trans 2003;31:83-6

Brouns MR, Matheson SF, Hu KQ, Delalle I, Caviness VS, Silver J, Bronson RT, Settleman J. The adhesion signaling molecule p190 RhoGAP is required for morphogenetic processes in neural development. Development 2000;127: 4891-903

Brouns MR, Matheson SF, Settleman J. p190 RhoGAP is the principal Src substrate in brain and regulates axon outgrowth, guidance and fasciculation. Nat Cell Biol 2001; 3:361-7
Chang JH, Gill S, Settleman J, Parsons SJ. c-Src regulates the simultaneous rearrangement of actin cytoskeleton, p190RhoGAP, and p120RasGAP following epidermal growth factor stimulation. J Cell Biol 1995;130:355-68

da Silva JS, Dotti CG. Breaking the neuronal sphere: regulation of the actin cytoskeleton in neuritogenesis. Nat Rev Neurosci 2002;3:694-704

Daniels RH, Hall PS, Bokoch GM. Membrane targeting of p21-activated kinase 1 (PAK1) induces neurite outgrowth from PC12 cells. EMBO J 1998;17:754-64

El-Benna J, Dang PMC, Gougerot-Pocidalo MA, Marie JC, Braut-Boucher F. p47phox, the phagocyte NADPH oxidase/NOX2 organizer: structure, phosphorylation and implication in diseases. EXP Mol Med 2009;41:217-25

Esposito D, Patel P, Stephens RM, Perez P, Chao MV, Kaplan $\mathrm{DR}$, Hempstead BL. The cytoplasmic and transmembrane domains of the $\mathrm{p} 75$ and Trk A receptors regulate high affinity binding to nerve growth factor. J Biol Chem 2001;276: 32687-95

Fincham VJ, Chudleigh A, Frame MC. Regulation of p190 Rho-GAP by v-Src is linked to cytoskeletal disruption during transformation. J Cell Sci 1999;112:947-56

Hadari YR, Kouhara H, Lax I, Schlessinger J. Binding of Shp2 tyrosine phosphatase to FRS2 is essential for fibroblast growth factor-induced PC12 cell differentiation. Mol Cell Biol 1998;18:3966-73

Harada A, Katoh H, Negishi M. Direct interaction of Rnd1 with FRS2 beta regulates Rnd1-induced down-regulation of RhoA activity and is involved in fibroblast growth factor-induced neurite outgrowth in PC12 cells. J Biol Chem 2005;280:18418-24

Haskell MD, Nickles AL, Agati JM, Su L, Dukes BD, Parsons SJ. Phosphorylation of p190 on Tyr1105 by c-Src is necessary but not sufficient for EGF-induced actin disassembly in C3H10T1/2 fibroblasts. J Cell Sci 2001; 114:1699-708

Hisata S, Sakisaka T, Baba T, Yamada T, Aoki K, Matsuda M, Takai Y. Rap1-PDZ-GEF1 interacts with a neurotrophin receptor at late endosomes, leading to sustained activation of Rap1 and ERK and neurite outgrowth. J Cell Biol 2007; 178:843-60

Jenei V, Deevi RK, Adams CA, Axelsson L, Hirst DG, Andersson T, Dib K. Nitric oxide produced in response to engagement of beta2 integrins on human neutrophils activates the monomeric GTPases Rap1 and Rap2 and promotes adhesion. J Biol Chem 2006;281:35008-20

Kao S, Jaiswal RK, Kolch W, Landreth GE. Identification of the mechanisms regulating the differential activation of the mapk cascade by epidermal growth factor and nerve growth factor in PC12 cells. J Biol Chem 2001;276:18169-77

Kim H, Hwang JS, Woo CH, Kim EY, Kim TH, Cho KJ, Seo $J M$, Lee SS, Kim JH. TNF- $\alpha$-induced up-regulation of intercellular adhesion molecule-1 is regulated by a Rac-ROS-dependent cascade in human airway epithelial cells. Exp Mol Med 2008;40:167-75

Kim JS, Diebold BA, Kim JI, Kim J, Lee JY, Park JB. Rho is 
involved in superoxide formation during phagocytosis of opsonized zymosans. J Biol Chem 2004;279:21589-97

Kozma R, Sarner S, Ahmed S, Lim L. Rho family GTPases and neuronal growth cone remodelling: relationship between increased complexity induced by Cdc42Hs, Rac1, and acetylcholine and collapse induced by RhoA and lysophosphatidic acid. Mol Cell Biol 1997;17:1201-11

Krugmann S, Williams R, Stephens L, Hawkins PT. ARAP3 is a PI3K- and rap-regulated GAP for RhoA. Curr Biol 2004;14:1380-4

Lad SP, Peterson DA, Bradshaw RA, Neet KE. Individual and combined effects of TrkA and p75NTR nerve growth factor receptors. A role for the high affinity receptor site. J Biol Chem 2003;278:24808-17

Luo L. Rho GTPases in neuronal morphogenesis. Nat Rev Neurosci 2000;1:173-80

Nusser N, Gosmanova E, Zheng Y, Tigyi G. Nerve growth factor signals through TrkA, phosphatidylinositol 3-kinase, and Rac1 to inactivate RhoA during the initiation of neuronal differentiation of PC12 cells. J Biol Chem 2002;277:35840-6

Ong SH, Guy GR, Hadari YR, Laks S, Gotoh N, Schlessinger J, Lax I. FRS2 proteins recruit intracellular signaling pathways by binding to diverse targets on fibroblast growth factor and nerve growth factor receptors. Mol Cell Biol 2000;20:979-89

Roskoski R Jr. Src kinase regulation by phosphorylation and dephosphorylation. Biochem Biophys Res Commun 2005; 331:1-14

Sofroniew MV, Howe CL, Mobley WC. Nerve growth factor signaling, neuroprotection, and neural repair. Annu Rev Neurosci 2001;24:1217-81
Vossler MR, Yao H, York RD, Pan MG, Rim CS, Stork PJ. cAMP activates MAP kinase and Elk-1 through a B-Raf- and Rap1-dependent pathway. Cell 1997;89:73-82

Welch HC, Condliffe AM, Milne LJ, Ferguson GJ, Hill K, Webb LM, Okkenhaug K, Coadwell WJ, Andrews SR, Thelen M, Jones GE, Hawkins PT, Stephens LR. P-Rex1 regulates neutrophil function. Curr Biol 2005;15:1867-73

Wu C, Lai CF, Mobley WC. Nerve growth factor activates persistent Rap1 signaling in endosomes. J Neurosci 2001; 21:5406-16

Yamada T, Sakisaka T, Hisata S, Baba T, Takai Y. RA-RhoGAP, Rap-activated Rho GTPase-activating protein implicated in neurite outgrowth through Rho. J Biol Chem 2005;280:33026-34

Yamaguchi Y, Katoh H, Yasui H, Mori K, Negishi M. RhoA inhibits the nerve growth factor-induced Rac1 activation through Rho-associated kinase-dependent pathway. J Biol Chem 2001;276:18977-83

Yasui $\mathrm{H}$, Katoh H, Yamaguchi Y, Aoki J, Fujita H, Mori K, Negishi M. Differential responses to nerve growth factor and epidermal growth factor in neurite outgrowth of PC12 cells are determined by Rac1 activation systems. J Biol Chem 2001;276:15298-305

York RD, Yao H, Dillon T, Ellig CL, Eckert SP, McCleskey EW, Stork PJ. Rap1 mediates sustained MAP kinase activation induced by nerve growth factor. Nature 1998;392: 622-6

Zhang SQ, Yang W, Kontaridis MI, Bivona TG, Wen G, Araki T, Luo J, Thompson JA, Schraven BL, Philips MR, Neel BG. Shp2 regulates SRC family kinase activity and Ras/Erk activation by controlling Csk recruitment. Mol Cell 2004; 13:341-55 